\title{
Nearest-neighbour distribution of distances in crystals from extended X-ray absorption fine structure
}

P. Fornasini, R. Grisenti, M. Dapiaggi, G. Agostini, and T. Miyanaga

Citation: The Journal of Chemical Physics 147, 044503 (2017);

View online: https://doi.org/10.1063/1.4995435

View Table of Contents: http://aip.scitation.org/toc/jcp/147/4

Published by the American Institute of Physics

\section{Articles you may be interested in}

Full-dimensional multi-state simulation of the photodissociation of thioanisole

The Journal of Chemical Physics 147, 044311 (2017); 10.1063/1.4994923

Image potential states from the van der Waals density functional

The Journal of Chemical Physics 147, 044708 (2017); 10.1063/1.4995441

Structural and dynamic characteristics in monolayer square ice

The Journal of Chemical Physics 147, 044706 (2017); 10.1063/1.4995432

Hydrogen bonding and vibrational energy relaxation of interfacial water: A full DFT molecular dynamics simulation

The Journal of Chemical Physics 147, 044707 (2017); 10.1063/1.4995437

Influence of sample preparation on the transformation of low-density to high-density amorphous ice: An explanation based on the potential energy landscape

The Journal of Chemical Physics 147, 044501 (2017); 10.1063/1.4993567

Drying and wetting transitions of a Lennard-Jones fluid: Simulations and density functional theory

The Journal of Chemical Physics 147, 044701 (2017); 10.1063/1.4993515

\section{AIP | The Joumal of \\ AIP| Chemical Physics}

PERSPECTIVES 


\title{
Nearest-neighbour distribution of distances in crystals from extended X-ray absorption fine structure
}

\author{
P. Fornasini, ${ }^{1, a)}$ R. Grisenti, ${ }^{1}$ M. Dapiaggi, ${ }^{2}$ G. Agostini, ${ }^{3, b)}$ and T. Miyanaga ${ }^{4}$ \\ ${ }^{1}$ Dipartimento di Fisica, Università di Trento, Via Sommarive 14, I-38123 Povo, Trento, Italy \\ ${ }^{2}$ Dipartimento di Scienze della Terra, Università di Milano, I-20133 Milano, Italy \\ ${ }^{3}$ ESRF, Grenoble, France \\ ${ }^{4}$ Department of Mathematics and Physics, Hirosaki University, Hirosaki, Aomori 036-8561, Japan
}

(Received 2 May 2017; accepted 10 July 2017; published online 28 July 2017)

\begin{abstract}
Extended X-ray absorption fine structure (EXAFS) is a powerful probe of the distribution of nearestneighbour distances around selected atomic species. We consider here the effect of vibrational disorder in crystals. The potential of EXAFS for the accurate evaluation of the coefficient of bond thermal expansion and its temperature dependence is discussed, with the aim of stimulating and facilitating the comparison with the results from total scattering experiments. The meaning of the distribution asymmetry in crystals and its connection with the effective potential anharmonicity and the bond expansion is quantitatively explored by comparing the results for a number of different systems. The extent of the relative atomic vibrations perpendicular to the bond direction and the perpendicular to parallel anisotropy are correlated with the extent of lattice negative thermal expansion as well as with the ionic mobility in superionic crystals. Published by AIP Publishing. [http://dx.doi.org/10.1063/1.4995435]
\end{abstract}

\section{INTRODUCTION}

Extended X-ray Absorption Fine Structure (EXAFS) is a widely employed probe of the local structure and dynamical behaviour of matter. ${ }^{1-3}$ Particularly appealing is the sensitivity of EXAFS to the distribution of distances between the atoms of the absorbing species and their nearest-neighbours. Gaussian distributions were considered in the first EXAFS applications. In 1979 Eisenberger and Brown ${ }^{4}$ demonstrated that the asymmetry of the distribution cannot be neglected if a reasonable evaluation of thermal expansion is sought. One possibility of going beyond the gaussian approximation is to express the distance distribution in terms of a restricted number of parameters that correspond to a peculiar physical model of the system..$^{5-8}$

A more general approach is based on the cumulant expansion. ${ }^{7,9-11}$ For relatively small degrees of thermal and structural disorder, the leading cumulants ${ }^{10}$ of the partial radial distribution $\rho(r)$ can be obtained with good approximation from the EXAFS analysis. ${ }^{12}$ The knowledge of the leading cumulants allows, in turn, the reconstruction of the distribution $\rho(r){ }^{7,12}$ More specific information is generally obtained by separately considering the values of the different cumulants and their variation with temperature ${ }^{13,14}$ or pressure, ${ }^{15,16}$ or the differences between different crystalline phases or non-crystalline forms of the same substance. ${ }^{17,18}$

Strengths and limitations of the cumulant method have been studied by various authors in relation with the extent of disorder and the shape of the distribution. ${ }^{7,12,19,20}$ The

\footnotetext{
a) Author to whom correspondence should be addressed: paolo.fornasini@ unitn.it

b) Present address: Leibniz-Institut für Katalyse e. V. an der Universität Rostock (LIKAT), Albert Einstein Str. 29a, 18059 Rostock, Germany.
}

main limitations are encountered when dealing with structural disorder. In liquid systems, the cumulant analysis can lead to reasonable fits to the experimental signals but to nearestneighbour distributions significantly different from the radial distributions measured by elastic scattering. ${ }^{21}$ A more refined analysis of EXAFS spectra for single-component liquids has been proposed and applied to a number of systems, ${ }^{20,22}$ where the starting point is a realistic model pair distribution function (from scattering experiments or from computer simulations) and the general constraints on the distributions for liquids are taken into account. An important achievement of this approach, enhanced by multiple scattering simulations, is the detection of icosahedral configurations in liquid copper. $^{23}$

In nano-particles, the distribution of bond distances is affected, in addition to the intrinsically anharmonic vibrational effects, by the variation of interatomic distances within a single nano-particle as well as by the distribution of nanoparticles sizes and shapes. ${ }^{24,25}$ A cumulant analysis of the first-shell EXAFS can help in disentangling the effects of thermal and structural disorder. ${ }^{26}$ Serious concerns have however been raised about the ability of the cumulant approach to fully characterise the radial distributions in nanoparticles. ${ }^{27,28}$ A general assessment on the problem is complicated by the large variety of systems, production methods, sizes and size distributions, shapes, as well as interactions with the support and with the environment which are encountered in practical applications.

More general solutions to the characterisation of the distance distributions in structurally disordered systems are based on more refined data analysis procedures, such as regularisation methods, ${ }^{29-31}$ on the support of molecular dynamics simulations, ${ }^{32-34}$ or on Reverse Monte Carlo (RMC) simulations. ${ }^{23,35,36}$ One advantage of such procedures is the 
possibility of simultaneously analysing the results of different experimental techniques.

In this paper, we focus our attention on the case of relatively weak vibrational disorder in simple crystals, for which a skillful application of the cumulant method can be considered highly reliable. It should be stressed that even for these relatively simple systems, a purely harmonic analysis of EXAFS spectra, in spite of its still widespread use, is in principle incorrect and can lead in many cases to non-negligible errors in nearest-neighbour distances. Original hints on local thermodynamical properties are obtained from temperature-dependent measurements. ${ }^{14,37}$ Checks of the experimental cumulants can be based on the agreement of their temperature dependence with theoretical expectations ${ }^{14,38}$ and on their reproduction by theoretical simulations. ${ }^{39-41}$

The sensitivity of EXAFS to the local structure of crystals, and in particular to the average nearest-neighbour distance (bond distance) and its thermal expansion, is shared by other correlation-sensitive techniques, such as the diffuse scattering of X-rays or neutrons. Bragg scattering from crystals is instead sensitive to the long range order and measures the distance between average atomic positions and the lattice thermal expansion, which are significantly smaller than the bond length and the bond expansion, respectively, owing to the effect of transverse vibrations. ${ }^{42-44}$ Recently, the potential of X-ray and neutron scattering for the study of the local structure of crystals has been enhanced by the development of the total scattering technique, ${ }^{45}$ which is based on the Fourier transform of the global signal made by Bragg peaks and diffuse scattering and thus contains information on the correlated short-range order.

The bond expansion has been measured by total scattering in framework structures ${ }^{46-50}$ as well as by EXAFS in diamond-zincblende structures, ${ }^{14,51,52}$ in framework structures ${ }^{53,54}$ and in delafossite structures. ${ }^{55}$ In those works, average values of the coefficient of bond thermal expansion have been estimated from linear fits to the temperature dependence of the bond length; by this procedure, however, the non-linear low-temperature behaviour is not evidenced, as is instead commonly done for the lattice expansion measured by Bragg diffraction or dilatometric techniques. ${ }^{56}$ Only recently a more refined evaluation of the temperature dependence of the bond expansion coefficient has been obtained from EXAFS data. ${ }^{57}$

The EXAFS cumulants can be expressed in terms of the mean square relative atomic displacements (MSRD). ${ }^{58}$ To first approximation, the second cumulant corresponds to the parallel MSRD. ${ }^{59}$ From the comparison of the bond lengths measured by EXAFS and Bragg scattering one can evaluate the perpendicular MSRD. ${ }^{38}$ The knowledge of both parallel and perpendicular MSRDs allows the reconstruction of the three dimensional distribution of vector distances between the absorbing and the backscattering atoms.

In spite of the widespread application of the cumulant method for the EXAFS study of a number of different systems, some points are still worth a deeper investigation, in order to extend the range of applications of the technique and to enhance the degree of accuracy of its results. (a) The nearest-neighbour distribution of distances has been found to be asymmetric even at relatively low temperatures in a number of crystals with different structural and thermodynamical properties; the distribution asymmetry is connected to the anharmonicity of the effective pair potential. A quantitative comparison between different systems can be made by different quantities that depend on the distribution asymmetry and on the potential anharmonicity, such as the temperature dependence of the third cumulant, the third-order force constant $k_{3}$, or the asymmetry coefficient of the distribution. These quantities are however not equivalent. Their use for quantitative comparisons can lead to apparently inconsistent results: for example, larger third cumulants generally correspond to smaller values of $\left|k_{3}\right|$.

(b) The relation between EXAFS and total scattering has been little explored up to now. ${ }^{4,60,61}$ Total scattering and EXAFS are complementary techniques: sensitivity to long range order and selectivity of the atomic species are the peculiar strengths of total scattering and EXAFS, respectively. The results from the two techniques have been mixed in RMC refinements. ${ }^{60,61}$ Total scattering and EXAFS are in general considered equivalent for the characterisation of the distribution of nearest-neighbour distances, in spite of the differences of underlying mechanisms of interaction of X-rays with matter, measurement apparatuses, and data analysis procedures. This equivalence is however not supported by a conclusive experimental evidence based on the separate study of the same model compound by the two techniques. In particular, it appears sensible to inquire into the relevance of taking into account the distribution asymmetry for obtaining accurate bond expansion values.

(c) The possibility of evaluating the perpendicular MSRD and the generally anisotropic three-dimensional distribution of vector distances has not yet been fully exploited. A correlation with the extent of lattice negative thermal expansion has been recently proposed ${ }^{62}$ and is worth a quantitative detailed study; besides, connections with other physical properties, such as ionic mobility and phase instabilities, are promising fields of investigation.

The aim of the present paper is to obtain a deeper understanding of the above points. By critically comparing the results of EXAFS studies of the nearest-neighbour distribution of distances in a number of relatively simple model systems, we gain new insights on the meaning of distribution asymmetry and effective potential anharmonicity as well as new hints for exploiting the information on the three-dimensional distribution of distances. The accurate characterisation of the nearest-neighbour distributions represents a contribution, from the EXAFS point of view, for a more refined comparison with total scattering.

The characterisation by EXAFS of the distance distributions due to weak vibrational disorder is shortly reviewed in Sec. II, and the main differences with total scattering are stressed. The possibility of directly reconstructing the 
distribution from the experimental cumulants is analysed in Sec. III. In Sec. IV, the information obtainable from the different cumulants is discussed; particular attention is given to the asymmetry of the distribution and to the anharmonicity of the effective pair potential. In Sec. V, the extent of perpendicular vibrations and the three-dimensional distribution of nearest-neighbour distances are discussed and correlated with the low-temperature lattice negative thermal expansion of tetrahedral semiconductors and other systems as well as with the ion mobility in the superionic crystals $\mathrm{AgI}$ and $\mathrm{CuCl}$. Section VI is dedicated to conclusions.

\section{EXAFS ANALYSIS}

The EXAFS function $\chi(k)$ is expressed in terms of the photo-electron wave-vector $k$, whose values typically span an interval from about 2 to about $20 \AA^{-1}$, corresponding to scattering vector values $Q=2 k$. The contribution of light scatterers is limited to low- $k$ values, while the contribution of heavy scatterers extends to higher $k$ values, in qualitative similarity with the behaviour of the X-ray atomic scattering factors.

The EXAFS function samples a partial radial distribution $\rho(r)$ of distances around the atoms of a given absorbing species, which is generally referred to as real distribution. Width and shape of $\rho(r)$ are determined by the relative atomic thermal vibrations and possibly by local structural disorder. In the following, we focus on systems where the contribution of structural disorder is negligible.

In the single scattering and plane-wave approximations, the contribution of each coordination shell to EXAFS is ${ }^{7}$

$$
\chi(k)=\frac{N S_{0}^{2}}{k} \operatorname{Im}\left\{f_{s}(k, \pi) e^{2 i \delta_{1}} \int_{0}^{\infty} P(r, \lambda) e^{2 i k r} d r\right\},
$$

where $N$ is the coordination number and $P(r, \lambda)$ $=\rho(r) \exp (-2 r / \lambda) / r^{2}$ is an effective distribution of distances. A simulated comparison between the real and effective distributions $\rho(r)$ and $P(r, \lambda)$ for a number of coordination shells of germanium (Fig. 1) clearly illustrates the short-range sensitivity of EXAFS, due to the spherical nature of the photoelectron probe and its short mean free path $\lambda \simeq 10 \AA$, to be contrasted by the long-range sensitivity of scattering experiments.

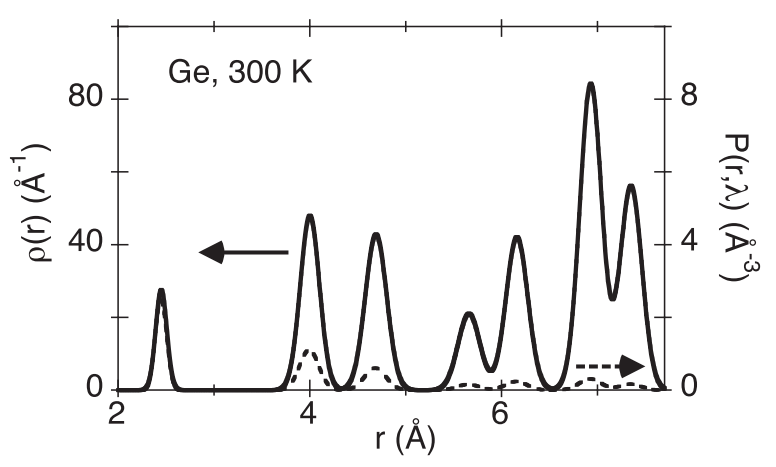

FIG. 1. Simulated distributions of distances for Ge at $300 \mathrm{~K}$ : real distribution $\rho(r)$ (continuous line, left scale) and effective distribution $P(r, \lambda)$ (dashed line, right scale). The widths of the first three peaks have been estimated from the experimental Debye-Waller factors of the first three coordination shells, ${ }^{63,64}$ respectively; for the remaining peaks, the experimental Debye-Waller factor of the third coordination shell has been considered. A mean free path $\lambda=8$ $\AA$ has been assumed.
In total scattering experiments, the pair correlation functions are directly obtained from the structure factors by a sine Fourier transform (FT). ${ }^{45,65}$ In EXAFS, the connection between the $k$ and $r$ spaces is made through a complex FT

$$
F(r)=\int_{k_{\min }}^{k_{\max }} \chi(k) W(k) k^{n} \exp (2 \mathrm{i} k r) d k,
$$

where $W(k)$ is a window function and $n$ a small integer number. The peaks of $|F(r)|$ correspond to the different coordination shells of the absorbing atom, while the real sine and cosine transforms give faster oscillations, owing to the relatively large value of $k_{\min } \simeq 2-3 \AA^{-1}$ (Ref. 6). The function $|F(r)|$ is different from the effective radial distribution $P(r, \lambda)$ for a number of reasons: the down-shift of about $0.2-0.3 \AA$ of the coordination shell peaks with respect to the true interatomic distances owing to the effect of the potential felt by the photoelectron, the multiple scattering contributions, the possible destructive interference between the contributions of different scattering paths, the FT artefacts.

If the first peak of $|F(r)|$ corresponds to only one distance and is sufficiently well separated from the other peaks, ${ }^{66}$ it can be back-transformed to single out the first-shell contribution. The resulting signal is affected by the artefacts introduced by both the direct and reverse FTs; these effects are however effectively eliminated by well established procedures of data analysis.

For weakly disordered systems, the amplitude and phase of the EXAFS function for one shell (from now on the first shell) can be parametrized in terms of the even and odd cumulants $C_{i}$ of the effective distribution $P(r, \lambda)$, respectively. ${ }^{10}$ The cumulants $C_{i}^{*}$ of the real distribution $\rho(r)$ are connected to the cumulants of the effective distribution $P(r, \lambda)$ by a simple recursion formula. ${ }^{67,68}$ The difference is generally significant only for the first cumulant ${ }^{10,69}$ and the corresponding transformation is now included in most data analysis packages. The first and second cumulants, $C_{1}^{*}=\langle r\rangle$ and $C_{2}^{*}=\sigma^{2}=\left\langle(r-\langle r\rangle)^{2}\right\rangle$, are the average value and the variance of the distribution, respectively. Higher order cumulants quantify the deviation of the distribution from a gaussian shape: in particular, the third cumulant $C_{3}^{*}=\left\langle(r-\langle r\rangle)^{3}\right\rangle$ is connected to the asymmetry.

The absolute values of the coordination number and of the leading cumulants can be evaluated by a non-linear fit of theoretically simulated spectra to experimental spectra, provided that the theoretical signal undergoes the same FTs of the experimental signal. ${ }^{70-74}$

The alternative ratio method ${ }^{10,12}$ eliminates the need for the theoretical input of $|f(k, \pi)|, \phi(k), S_{0}^{2}$, and $\lambda(k)$ and cancels the FT artefacts but only gives the values of the coordination number and of the leading cumulants with respect to a given reference. Absolute values of the second and third cumulant can anyway be evaluated by fitting to theoretical models. ${ }^{37}$

The relative merits of the two methods, non-linear fit and ratio-method, are discussed in Ref. 68. The accuracy of theoretical calculations influences the accuracy of the final parameters: uncertainties of the order of $0.01 \AA$ are typically expected for absolute bond distances. Much smaller 
uncertainties, less than $0.001 \AA$, can be obtained for relative values. ${ }^{38,63}$

\section{THE RADIAL DISTRIBUTION}

Once the leading cumulants have been obtained from the EXAFS analysis, the characteristic function of the real distribution $\rho(r)$ can be evaluated in a convenient interval centred on $k=0$. The distribution $\rho(r)$ can then be recovered by Fourier transforming the characteristic function. ${ }^{7}$ Such a procedure was applied to a number of simple systems such as $\beta$ - $\mathrm{AgI}^{12,75}$ (see below, Sec. V C), $\mathrm{Cu},{ }^{38} \mathrm{Ge},{ }^{76} \mathrm{CdSe} .{ }^{77}$ For amorphous $\mathrm{Ge},{ }^{78}$ not only the distribution reconstructed from cumulants at $300 \mathrm{~K}$ was found in agreement with the $\Gamma$-like distribution of Filipponi and Di Cicco, ${ }^{8}$ but also its tiny variations induced by hydrogenation could be detected.

As an example, the distributions $\rho(r)$ of the Cd-Te distance in $\mathrm{CdTe}$, reconstructed at different temperatures, are shown in Fig. 2, left. The increase with temperature of the width and asymmetry of the distribution is evident. The mean value of the distribution increases by $0.012 \AA$ from 5 to $300 \mathrm{~K}$.

In Fig. 2, right, the asymmetric distribution at $300 \mathrm{~K}$ reconstructed from the first four EXAFS cumulants (continuous line) is compared with the symmetric distribution reconstructed from a purely harmonic analysis of the EXAFS signal (dashed line). The visual difference between the two distributions is quite small; however, the difference between their mean values, 2.812 and $2.800 \AA$, is non negligible for many purposes and is by far better evidenced in the plot of phase differences (see below, Fig. 5); for the gaussian distribution, the mean is equal to the mode, and for the asymmetric distribution the mean is larger than the mode. When a gaussian function is fitted to the asymmetric distribution, one obtains an average value $2.805 \AA$, intermediate between the values from the gaussian and the asymmetric analysis of EXAFS.

While the inclusion of asymmetry in EXAFS analyses is now a standard procedure, the nearest-neighbour distribution is generally fitted to a gaussian shape in the analysis of the pair distribution functions (PDF) from total scattering. ${ }^{46,48}$ In Ref. 60, the comparison between EXAFS and total

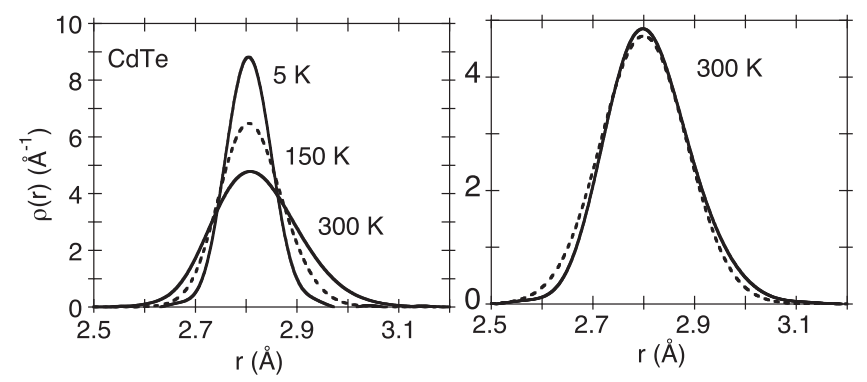

FIG. 2. Left: real distributions of nearest-neighbour distances in CdTe at various temperatures reconstructed from the first four EXAFS cumulants. Right: real distributions at $300 \mathrm{~K}$ reconstructed from the first four cumulants (continuous line) and from the first two cumulants obtained neglecting higher order cumulants in the analysis (dashed line). [Adapted from Fig. 3 of P. Fornasini and R. Grisenti, J. Chem. Phys. 141, 164503 (2014). Copyright 2014 AIP Publishing LLC.] scattering for the Ni model compound was made assuming a gaussian distribution too. In recent experiments on $\mathrm{M}_{2} \mathrm{O}$ crystals ( $\mathrm{M}=\mathrm{Cu}, \mathrm{Ag}$, cuprite structure), a reasonable agreement was found between the $\mathrm{M}-\mathrm{O}$ bond expansions measured by EXAFS $^{53}$ (asymmetric distribution) and total scattering ${ }^{49}$ (symmetric distribution), while a significant disagreement was found between two different total scattering experiments. ${ }^{49,50}$ No definitive conclusions can be drawn from these few examples, in view of the relatively low quality of the EXAFS data on cuprites with respect to the data here presented on tetrahedral semiconductors. It is anyway evident that the comparison of the distributions and of their parameters measured by different techniques is still an open problem. In principle, an advantage of EXAFS is the possibility of an effective elimination of the Fourier transform artefacts.

Finally, it is worth noting that the possibility of reconstructing the distribution from the experimental EXAFS cumulants depends on the convergence properties of the cumulant series, which could be critical even for purely vibrational effects. ${ }^{7,12}$ In some cases, the more refined splice method can be useful: ${ }^{7}$ the experimental cumulants are used to reconstruct only the low- $k$ missing part of the signal; the reconstructed portion is then spliced to the experimental signal and to its specular image for negative $k$ values. The Fourier transform of the characteristic function so obtained from $-k_{\max }$ to $+k_{\max }$ allows the evaluation of the distribution of distances. ${ }^{79-81}$

\section{INFORMATION FROM CUMULANTS}

More detailed information is obtained by separately considering the different cumulants of a given system and possibly their temperature and/or pressure dependence. By comparing the cumulants of different systems, one can get further information not only on the physical properties of the systems but also on the very meaning of cumulants.

The results for copper and for some semiconductors with the zincblende or wurtzite structure (tetrahedral coordination) are summarised in Table I. The results for $\mathrm{Cu}, \mathrm{Ge}, \mathrm{GaAs}$, and $\mathrm{CdTe}$, characterised by a higher degree of accuracy, are shown in some figures; the comparison between these four systems is particularly significant because the ratio of atomic masses in the binary compounds GaAs and CdTe is nearly one (1.07 and 1.13 , respectively).

\section{A. Bond thermal expansion}

In Fig. 3, the relative percent bond expansions measured by the first EXAFS cumulant for $\mathrm{Cu},{ }^{38} \mathrm{Ge},{ }^{14,63} \mathrm{GaAs},{ }^{52}$ and $\mathrm{CdTe}^{51,82}$ (full circles) are compared with the relative percent lattice expansions from Bragg diffraction or from dilatometric measurements (continuous lines). In the simple systems here considered, the lattice parameter is proportional to the distance $R_{c}$ between the average atomic positions.

For the tetrahedral semiconductors here considered, the lattice parameters are characterised by a negative thermal expansion (NTE) in a limited low-temperature interval, ${ }^{83}$ whose extent increases with increasing ionicity. For all systems, the bond expansion measured by EXAFS is positive in 
TABLE I. Results of the first-shell EXAFS analysis for copper and for some tetrahedral semiconductors, listed in order of increasing Phillips ionicity.

\begin{tabular}{|c|c|c|c|c|c|c|c|c|c|c|c|}
\hline & Ion. & $\begin{array}{c}v_{E} \\
(\mathrm{THz})\end{array}$ & $\begin{array}{c}k_{0}=k_{\|} \\
\left(\mathrm{eV} / \AA^{2}\right)\end{array}$ & $\begin{array}{c}C_{3} \\
\left(10^{-4} \AA^{3}\right) \\
(300 \mathrm{~K})\end{array}$ & $\begin{array}{c}k_{3} \\
\left(\mathrm{eV} / \AA^{3}\right)\end{array}$ & $\begin{array}{c}-6 k_{3} / k_{0}^{3 / 2} \\
\left(\mathrm{eV}^{-1 / 2}\right)\end{array}$ & $\begin{array}{c}-k_{3} / k_{0} \\
\left(\AA^{-1}\right)\end{array}$ & $\begin{array}{c}k_{\perp} \\
\left(\mathrm{eV} / \AA^{2}\right)\end{array}$ & $\left(k_{\|} / k_{\perp}\right)^{1 / 2}$ & $\begin{array}{c}\sigma_{\|} /\langle r\rangle \\
(\%) \\
(300 \mathrm{~K})\end{array}$ & $\begin{array}{c}\sigma_{\perp} /\langle r\rangle \\
(\%) \\
(300 \mathrm{~K})\end{array}$ \\
\hline $\mathrm{Cu}^{38}$ & 0 & 4.96 & 3.2 & 1.5 & -1.37 & 1.44 & 0.43 & 2.72 & 1.08 & 3.6 & 3.8 \\
\hline $\mathrm{Ge}^{14,64}$ & 0 & 7.7 & 8.5 & 0.32 & -4.31 & 1.04 & 0.51 & 2.89 & 1.71 & 2.4 & 3.9 \\
\hline $\mathrm{GaAs}^{52}$ & 0.31 & 6.86 & 6.97 & 0.46 & -3.94 & 1.28 & 0.56 & 1.85 & 1.94 & 2.6 & 4.8 \\
\hline $\mathrm{InP}^{18}$ & 0.42 & 8.21 & 6.69 & 1.0 & -7.5 & 2.6 & 1.12 & 1.10 & 2.46 & 2.6 & 5.9 \\
\hline $\mathrm{CdSe}^{77}$ & 0.7 & 4.77 & 4.32 & 1.4 & -2.8 & 1.87 & 0.65 & 0.96 & 2.12 & 2.9 & 6.3 \\
\hline $\mathrm{CdTe}^{51}$ & 0.717 & 3.92 & 3.76 & 1.47 & -2.05 & 1.69 & 0.54 & 0.80 & 2.17 & 3.1 & 6.3 \\
\hline $\mathrm{CuCl}^{68}$ & 0.746 & 3.93 & 1.40 & 15. & -1.23 & 4.45 & 0.88 & 0.26 & 2.32 & 5.8 & 13.5 \\
\hline$\beta-\mathrm{AgI}^{75}$ & 0.77 & 2.71 & 1.75 & 5.6 & -0.75 & 1.94 & 0.43 & 0.6 & 1.71 & 4.3 & 7.3 \\
\hline
\end{tabular}

the full temperature range and always larger than the lattice expansion. This behaviour is not unexpected, due to the effect of perpendicular vibrations, ${ }^{42,43,84}$ and had been experimentally anticipated by pioneering EXAFS and total scattering measurements. ${ }^{46,75}$

The lattice expansion is the result of two contributions, a positive one due to the anharmonicity of the effective pair potential (bond expansion) and a negative one due to tension effects; ${ }^{44,85}$ a crystal exhibits NTE when tension effects prevail over bond expansion. As one can see in Fig. 3, the increase of the ionicity and of the NTE strength is accompanied by an increase of the positive bond expansion. Actually, when the ionicity increases, the rigidity of the bond angles is reduced and the relative perpendicular vibrations are facilitated; as a consequence of the larger perpendicular vibrations, more room is made available even for longitudinal vibrations and thus for bond expansion.

The negative contribution of tension effects can be obtained by subtracting the bond expansion $\delta\langle r\rangle$ measured by EXAFS from the crystallographic expansion $\delta R_{c}{ }^{57}$ A coefficient of bond expansion can be defined in analogy with the coefficient of lattice expansion,

$$
\alpha_{\text {bond }}(T)=\frac{1}{\langle r\rangle}\left(\frac{\partial\langle r\rangle}{\partial T}\right)_{p} .
$$

The temperature dependence of $\alpha_{\text {bond }}(T)$ has been recently evaluated by the direct application of (3) to the expansion data of $\mathrm{Cu}$ and CdTe. ${ }^{57}$ Similarly, by differentiating the tension contribution with respect to $T$, a coefficient of tension $\alpha_{\text {tens }}(T)$ has also been evaluated. The two coefficients $\alpha_{\text {bond }}(T)$ and $\alpha_{\text {tens }}(T)$ for $\mathrm{Cu}$ and $\mathrm{CdTe}$ are compared with the lattice coefficient $\alpha_{\text {cryst }}$ in Fig. 4.

The lattice expansion (solid symbols in Fig. 4) is negative when $-\alpha_{\text {tens }}$ prevails over $\alpha_{\text {bond }}$ : this happens below about 70 $\mathrm{K}$ for $\mathrm{CdTe}$, never for $\mathrm{Cu}$. The tension effect is stronger for $\mathrm{CdTe}$, weaker for $\mathrm{Cu}$, where perpendicular vibrations are anyway not negligible. ${ }^{38}$ It is interesting to compare this local approach with the thermodynamic approach, where the sign of the lattice expansion is given, within the quasi-harmonic approximation, by the weighted sum of the normal mode Grüneisen parameters. ${ }^{87}$ Negative mode Grüneisen parameters have been calculated for transverse acoustic modes of $\mathrm{CdTe},{ }^{88}$ no negative mode Grüneisen parameters have been found for $\mathrm{Cu} .{ }^{89,90}$

Tension effects are not necessarily due to normal modes with negative Grüneisen parameters. For a closed-packed structure, such as the fcc one of $\mathrm{Cu}$, one can expect that a given normal mode gives rise to both parallel and perpendicular relative vibrations for two different orientations of the nearest-neighbour vector distance, respectively.

\section{B. Distribution variance}

The second cumulant, or EXAFS Debye-Waller exponent, is the variance $\sigma^{2}$ of the distribution $\rho(r)$. Its temperature dependence can generally be fitted by an Einstein model. ${ }^{37}$ To the Einstein frequency $v_{E}$, an effective force constant is associated, $k_{0}=4 \pi^{2} \mu v_{E}^{2}$, which measures the strength of the interaction between the nearest-neighbour pair embedded within the entire system. The nearest-neighbour Einstein
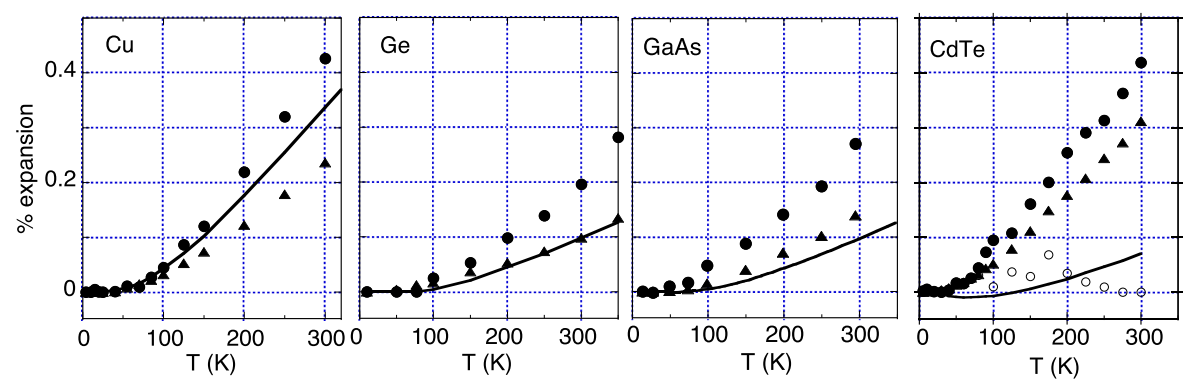

FIG. 3. Relative percent bond expansions of $\mathrm{Cu}, \mathrm{Ge}, \mathrm{GaAs}$, and $\mathrm{CdTe}$. The solid circles are the EXAFS values $\delta\langle r\rangle$, the continuous lines are the values $\delta R_{c}$ proportional to the lattice expansion. The triangles are the contributions to the bond expansion solely due to the asymmetry of the distribution. The open circles in the right panel are the bond expansion values obtained by a purely harmonic analysis of the CdTe EXAFS signals. 


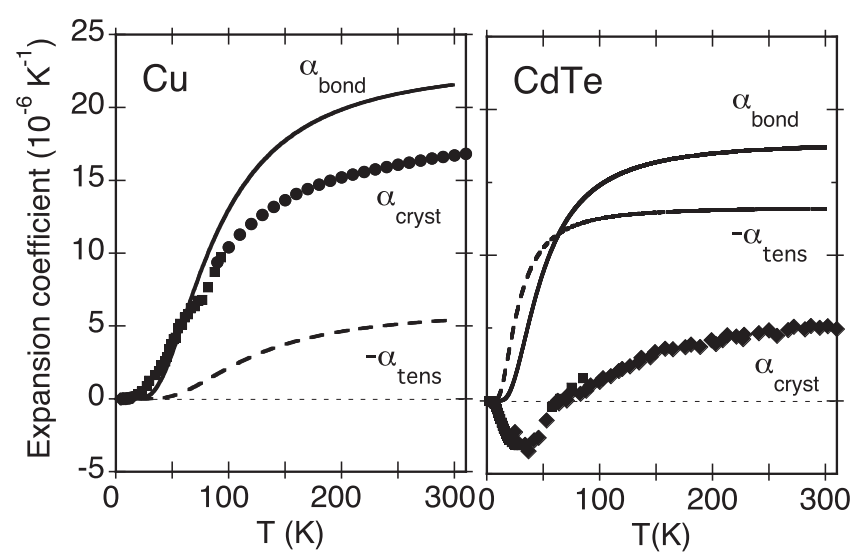

FIG. 4. Coefficient of bond thermal expansion $\alpha_{\text {bond }}$ (continuous lines) evaluated from the first EXAFS cumulants of $\mathrm{Cu}$ (left) and CdTe (right). The solid symbols give the coefficients of lattice expansion $\alpha_{\text {cryst }}$ of $\mathrm{Cu}^{56}$ and $\mathrm{CdTe}{ }^{83,86}$ The dashed line is the opposite of the coefficient of negative expansion $\alpha_{\text {tens }}$ due to the tension effects. [Adapted from Figs. 2 and 4 of P. Fornasini and R. Grisenti, J. Chem. Phys. 141, 164503 (2014). Copyright 2014 AIP Publishing LLC.]

frequencies and the reduced force constants are listed in Table I for a number of systems. For the tetrahedral semiconductors listed in Table I, the force constant $k_{0}$ decreases with increasing ionicity. The Einstein frequency, which measures the response to the interaction forces, depends on the inertia, measured by the reduced mass; that is why, for example, the Einstein frequency of InP is larger than the Einstein frequency of Ge.

At high temperatures, the Einstein model approaches the classical value $\sigma_{\infty}^{2}=k_{B} T / k_{0}$, independent of mass. For $T \rightarrow 0$, however, $\sigma_{E}^{2} \rightarrow \hbar / 2\left(\mu k_{0}\right)^{1 / 2}$ and depends on the reduced mass; the isotopic effect on the EXAFS Debye-Waller factor of Ge has been experimentally detected. ${ }^{63}$

\section{Distribution asymmetry}

The third cumulant, which depends on the asymmetry of the distribution $\rho(r)$, is important for two reasons. First, its neglect leads to wrong values of bond distances and bond expansions. Second, its temperature dependence gives interesting clues on the local dynamical behaviour.

The ratio method allows a visual evaluation of the influence of the third cumulant on the values of the first cumulant. For example, Fig. 5 shows the phase difference between $300 \mathrm{~K}$ and $20 \mathrm{~K}$ in CdTe, divided by $2 k$ and plotted against $k^{2}$, for a number of different independent measurements. According to the ratio method,

$$
\frac{\Phi^{(s)}-\Phi^{(m)}}{2 k}=\left[C_{1}^{(s)}-C_{1}^{(m)}\right]-\frac{4}{3}\left[C_{3}^{(s)}-C_{3}^{(m)}\right] k^{2},
$$

where sample $s$ and model $m$ correspond here to $300 \mathrm{~K}$ and $20 \mathrm{~K}$, respectively. The intercept and the slope of the straight line best fitting (4) give the variation of the first and of the third cumulants, respectively, of the effective distribution.

The intercept of the oblique dashed line in Fig. 5 gives a first cumulant variation, between 20 and $300 \mathrm{~K}$, of $+0.0075 \AA$ for the effective distribution, corresponding to $+0.012 \AA$ for the real distribution. Neglecting the third cumulant corresponds to fitting the phase difference with the horizontal dotted line in

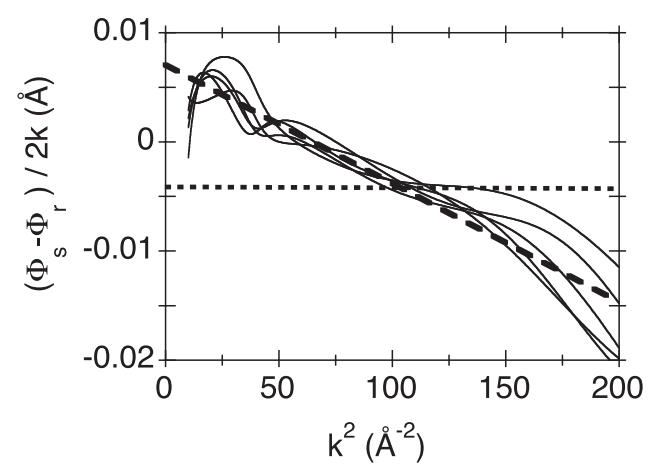

FIG. 5. Plot of phase differences $[\Phi(300 \mathrm{~K})-\Phi(20 \mathrm{~K})] / 2 k$ versus $k^{2}$ for different independent measurements at the Te $\mathrm{K}$ edge of CdTe. The oblique dashed line is the best fitting function $C_{1}-4 C_{3} k / 3$, the horizontal dotted line is the best fitting constant function $C_{1}$. [Adapted from Fig. 3 of P. Fornasini and R. Grisenti, J. Chem. Phys. 141, 164503 (2014). Copyright 2014 AIP Publishing LLC.]

Fig. 5; this procedure would lead to first-cumulant variations of $\simeq-0.0045 \AA$ and $\simeq-0.0001 \AA$ for the effective and real distributions, respectively. The gaussian approximation thus underestimates the real bond expansion at $300 \mathrm{~K}$ in CdTe by about $0.012 \AA$. The inadequacy of the gaussian approximation is also evident in the right panel of Fig. 3, where the open circles represent the bond thermal expansion of CdTe obtained when the third cumulant is neglected. Not only an exceedingly small expansion is obtained but also the spread of values is larger than that obtained when the third cumulant is included in the analysis.

The third EXAFS cumulants of $\mathrm{Cu}, \mathrm{Ge}, \mathrm{GaAs}$, and CdTe (edge $\mathrm{Cd} \mathrm{K}$ ) are shown in the top row of Fig. 6. Their temperature dependence is consistent with the quantum perturbative model $^{91,92}$

$$
C_{3} \simeq C_{3}^{*} \simeq-\frac{2 k_{3} \sigma_{0}^{4}}{k_{0}} \frac{z^{2}+10 z+1}{(1-z)^{2}}
$$

where $z=\exp \left(-\beta h v_{E}\right)$ and $\sigma_{0}^{2}=h /\left(4 \pi^{2} v_{E} \mu\right)$. By fitting (5) to the experimental values of the third cumulant, the force constants $k_{3}$ listed in Table I have been obtained.

At a given temperature, the third cumulant of the tetrahedral semiconductors here considered increases with the increase of ionicity, from Ge to CdTe; the values of $C_{3}$ at $300 \mathrm{~K}$ are listed in Table I; the third cumulant of copper is comparable with the third cumulant of CdTe. However, the modulus of $k_{3}$ decreases when the ionicity increases in semiconductors (with the exception of InP) and is larger for $\mathrm{Ge}$ than for $\mathrm{Cu}$. This apparent inconsistency can be explained by considering that, according to (5), the third cumulant depends on both $k_{3}$ and $k_{0}$ and that, according to Table I, the decrease of $\left|k_{3}\right|$ is accompanied by a decrease of $k_{0}$.

The degree of asymmetry of a distribution is better measured by the ratio $C_{3} / C_{2}^{3 / 2}$ (asymmetry or skewness parameter). ${ }^{9}$ The asymmetry parameters plotted in the bottom row of Fig. 6 as a function of temperature reveal that the asymmetry of the distribution is not so different for the different systems, in spite of the differences of $k_{0}$ and $k_{3}$.

At high temperature, the classical approximation for cumulants $^{13,21}$ gives, to first order, 

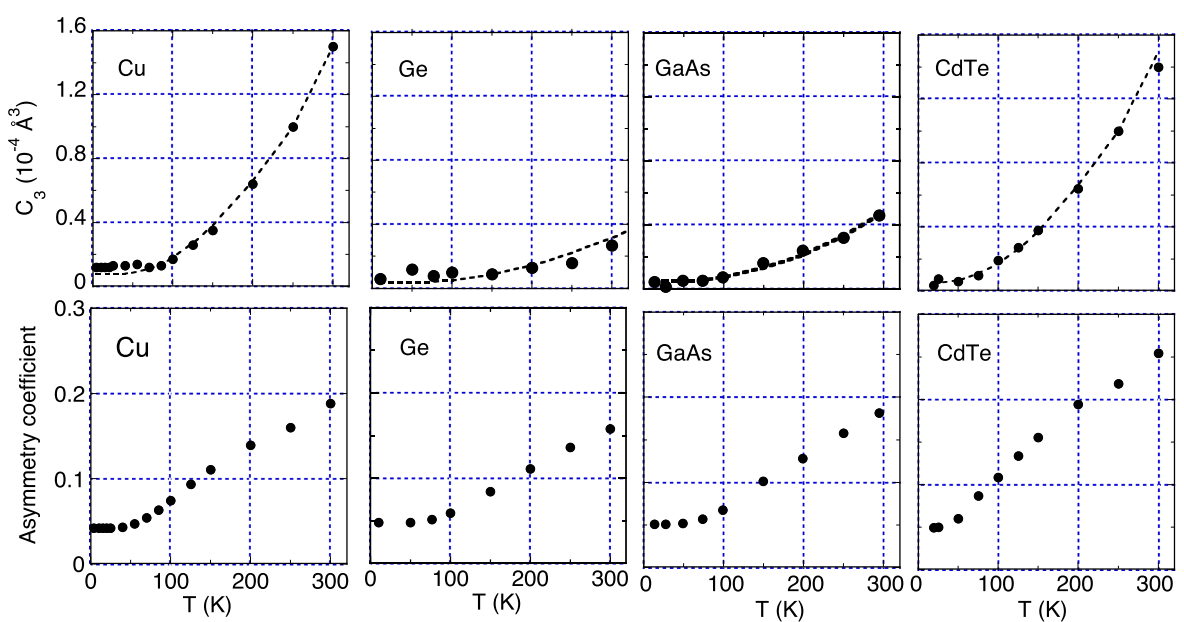

FIG. 6. Top row: third cumulant of the nearest-neighbour distribution of distances for $\mathrm{Cu}, \mathrm{Ge}, \mathrm{GaAs}$, and $\mathrm{CdTe}$. The solid circles are the EXAFS values, the dashed lines are the quantum perturbative model (5). Bottom row: corresponding asymmetry parameters $C_{3} / C_{2}^{3 / 2}$.

$$
C_{2}(T)=\frac{k_{B} T}{k_{0}}, \quad C_{3}(T)=-\frac{6 k_{3}}{k_{0}^{3}}\left(k_{B} T\right)^{2},
$$

whence one gets the temperature dependence of the asymmetry parameter

$$
\frac{C_{3}}{C_{2}^{3 / 2}}=-6 \frac{k_{3}}{k_{0}^{3 / 2}}\left(k_{B} T\right)^{1 / 2} .
$$

The values of $-6 k_{3} / k_{0}^{3 / 2}$ are listed in Table I. One can observe a correlation of the asymmetry parameter with the ionicity and the atomic mass ratio. For atomic mass ratios not larger than 1.2 (Ge, GaAs, CdTe, AgI), the asymmetry increases with ionicity. The deviation from this trend observed for InP, CdSe, and $\mathrm{CuCl}$ can be connected to the larger mass ratios $(3.7,1.42$, and 1.79 , respectively): the vibrational motion of the lighter atom is more intense and can be expected to give rise to larger asymmetric contributions.

\section{Effective pair potential}

Once the lowest order effective force constants have been determined from the leading experimental cumulants, one can calculate the effective potential (energy)

$$
V(x)=\frac{1}{2} k_{0} x^{2}+k_{3} x^{3}+k_{4} x^{4}+\cdots,
$$

where $x=r-r_{0}$ is the deviation of the interatomic distance from the position of the potential minimum.

The effective potentials calculated from the first term (harmonic approximation) and from the first two terms of (8) are shown in Fig. 7 as dashed and continuous lines, respectively. The effective force constants $k_{0}$ and $k_{3}$ characterize the harmonic and the first anharmonic contributions, respectively. The ratio between the contribution to $V(x)$ of the 3rd-order anharmonic and the harmonic contributions is proportional to the displacement $u$; the proportionality constant $k_{3} / k_{0}$ can be assumed as a measure of the anharmonicity of the effective potential (if higher orders are neglected). The values of $k_{3} / k_{0}$ are listed in Table I: they are quite similar for all systems, with the exception of $\mathrm{InP}$ and $\mathrm{CuCl}$.

Only for diatomic molecular gases does the effective potential $V(x)$ correspond to the single pair potential and the bond expansion can be accounted for solely by the anharmonicity of the single pair potential; according to Frenkel and Rehr, ${ }^{91}$ the net expansion $a(T)$ due to the potential anharmonicity is to first order

$$
a(T)=\langle r\rangle-r_{0} \simeq-\left(3 k_{3} / k_{0}\right) C_{2}^{*}(T) .
$$

The bond thermal expansion is a joint effect of anharmonicity, measured by the ratio $k_{3} / k_{0}$, and of the amplitude of relative vibrations, measured by the second cumulant. It is worth noting that different effects of anharmonicity, such as the bond thermal expansion and the asymmetry of the distribution of distances, differently depend on the potential anharmonicity measured by the ratio $k_{3} / k_{0}$. For example, Ge and $\mathrm{CdTe}$ have a very similar value of $k_{3} / k_{0}$; the distribution asymmetry is instead quite different and the bond expansion is very different.

For many-atomic systems, the distribution $\rho(r)$ can be affected by both vibrational and structural disorder. Even when only vibrational disorder is present, the effective potential $V(r)$ does not correspond to the single pair potential, since it depends on the statistically averaged behaviour of all the atoms in the crystal. ${ }^{93}$ Experimental results ${ }^{38,75,94}$ show that the bond expansion can be completely accounted for only if a temperature-dependent shift of the minimum position of the effective pair potential is considered, so that ${ }^{52}$
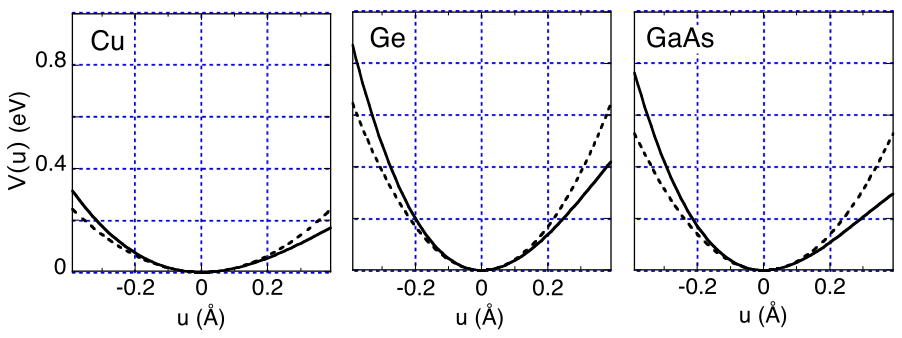

FIG. 7. Effective potentials calculated according to (8). Dashed lines are the harmonic potentials (only $k_{0}$ taken into account). Continuous lines include the first anharmonic term proportional to $k_{3}$. 


$$
\langle r\rangle \simeq r_{0}+a(T)+r_{v}(T)
$$

where $r_{v}$ is the contribution due to the shift of the effective potential minimum.

For all measured systems, the expansion $\delta a(T)=a(T)$ $-a(0)$ due to asymmetry (triangles in Fig. 3) has been found smaller than the bond expansion $\delta\langle r\rangle$, indicating that $r_{v}(T)>$ 0 . According to Fig. 3, the expansion $\delta a(T)$ is smaller than the crystallographic expansion $\delta R_{c}$ for $\mathrm{Cu}$, it is equal for $\mathrm{Ge}$ and larger for $\mathrm{GaAs}$ and $\mathrm{CdTe}$, the difference increasing with increasing ionicity.

The experimental values of bond thermal expansion and of third cumulant measured by EXAFS have been reproduced for $\mathrm{Cu}$ by path-integral Monte-Carlo simulations ${ }^{39,95}$ and for Ge and CdSe by Molecular Dynamics simulations. ${ }^{41,96}$ The simulations confirm the shift of the effective potential minimum.

The relation between single pair, effective pair, and single atom potentials has been investigated for copper and nickel, ${ }^{97}$ assuming a single-pair Morse potential and taking into account the influence of all the nearest neighbours of the absorber and backscatterer atoms following the approach of Van Hung and Rehr. ${ }^{98}$ The effective pair potential maintains memory of the anharmonicity of the Morse potential, although reduced with respect to the single pair potential; the harmonic approximation is instead good for the single-atom potential. This result suggests some caution in applying to the relative pair vibrations the same approximations that are common for single atom vibrations, such as the quasi-harmonic approximation. ${ }^{43}$

Finally, it is worth remembering that the anharmonicity of the effective one-dimensional pair potential is connected in a complicated way to the anharmonicity of the crystal potential, which is defined in terms of the Born-von Karman expansion in a $3 N$-dimensional configuration space. For example, one can show that a perfectly harmonic potential acting between all atomic pairs would give rise to an anharmonic crystal potential with a negative lattice expansion. ${ }^{99}$

\section{THREE-DIMENSIONAL DISTRIBUTION}

Further insights can be gained if the EXAFS cumulants are expressed in terms of the atomic displacements with respect to the average atomic positions. In particular, one can compare the bond distance measured by EXAFS with the corresponding crystallographic distance and one can reconstruct the threedimensional distribution of vector distances.

The instantaneous inter-atomic vector distance $\vec{r}$ is

$$
\vec{r}=\vec{R}_{c}+\Delta \vec{u},
$$

where $\vec{R}_{c}$ is the distance between the average atomic positions and $\Delta \vec{u}=\vec{u}_{b}-\vec{u}_{a}$ is the displacement of the backscatterer atom with respect to the absorber atom. Note that $\vec{R}_{c}$ includes the crystallographic thermal expansion and only for a perfectly harmonic crystal corresponds to the distance between the atomic positions at the minimum of the crystal potential. ${ }^{100}$ It is further convenient to separately consider the projections $\Delta u_{\|}$and $\Delta u_{\perp}$ along the $\hat{R}_{c}$ direction and in the perpendicular plane, respectively. ${ }^{58}$

By expanding the expression of the instantaneous scalar distance $r=\left[\left(\vec{R}_{c}+\Delta \vec{u}\right)^{2}\right]^{1 / 2}$ and taking into account that $\left\langle\Delta u_{\|}\right\rangle=\left\langle\left(\hat{R} \cdot \vec{u}_{b}\right)\right\rangle+\left\langle\left(\hat{R} \cdot \vec{u}_{a}\right)\right\rangle=0$ because the atomic oscillations are around the average positions, one finds the firstorder relation between bond distance and crystallographic distance $^{38,43}$

$$
\langle r\rangle \simeq R_{c}+\left\langle\Delta u_{\perp}^{2}\right\rangle / 2 R_{c} .
$$

By subtracting the crystallographic expansion $\delta R_{c}$ from the bond expansion $\delta\langle r\rangle$ measured by EXAFS (see Fig. 3), one obtains the temperature variation $\delta\left\langle\Delta u_{\perp}^{2}\right\rangle$ of the perpendicular MSRD. Absolute values $\left\langle\Delta u_{\perp}^{2}\right\rangle$ can be evaluated by fitting to an Einstein model. ${ }^{101}$

The second cumulant is ${ }^{102}$

$$
\begin{aligned}
C_{2} & =\sigma^{2}=\left\langle(r-\langle r\rangle)^{2}\right\rangle \\
& \simeq\left\langle\Delta u_{\|}^{2}\right\rangle+\frac{1}{4 R_{c}^{2}}\left\{\left\langle\Delta u_{\perp}^{4}\right\rangle-\left[\left\langle\Delta u_{\perp}^{2}\right\rangle\right]^{2}\right\},
\end{aligned}
$$

where the dominant contribution is the parallel mean square relative displacements (MSRD) $\left\langle\Delta u_{\|}^{2}\right\rangle$, which is in turn the sum of the independent mean square displacements of the two atoms, plus a correlation term, generally negative. ${ }^{59}$ The parallel MSRD can be directly obtained from total scattering experiments too. ${ }^{60,103}$

The parallel and half the perpendicular MSRDs of some systems are shown in Fig. 8. The Einstein model best-fitting the temperature dependence of the perpendicular MSRD ${ }^{101}$ allows one to evaluate the effective perpendicular force constant $k_{\perp}$ (Table I), which measures the resistance against bond bending (not to be confused with the force constants of lattice dynamical models ${ }^{52}$ ). For the tetrahedral semiconductors, the parallel force constants $k_{\|}=k_{0}$ are larger than the perpendicular force constants $k_{\perp}$; both quantities decrease when the ionicity increases. For copper, the difference between parallel and perpendicular force constants is quite small.

\section{A. Anisotropy of relative atomic vibrations}

The knowledge of both parallel and perpendicular MSRDs allows the reconstruction of a three-dimensional ellipsoidal

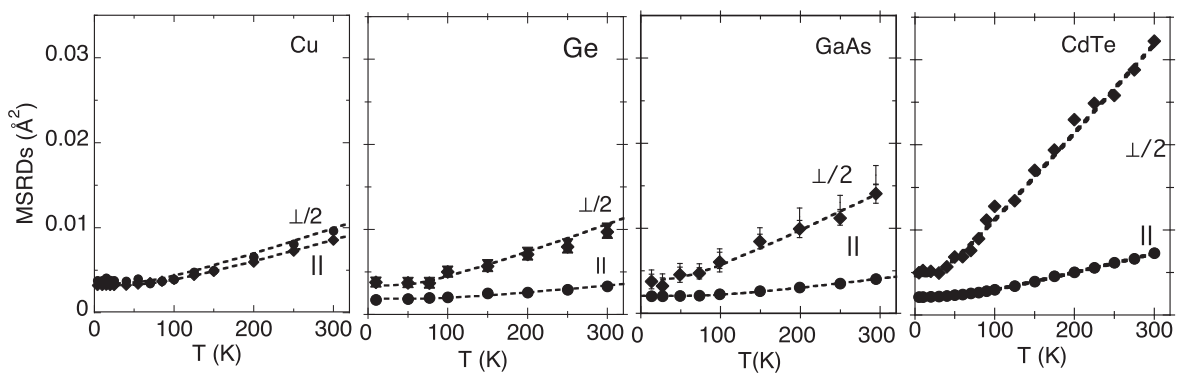

FIG. 8. Parallel and halved perpendicular MSDRs, $\left\langle\Delta u_{\|}^{2}\right\rangle$ (solid circles) and $\left\langle\Delta u_{\perp}^{2}\right\rangle / 2$ (solid diamonds), respectively, for $\mathrm{Cu}, \mathrm{Ge}, \mathrm{GaAs}$ (edge $\mathrm{Ga}$ ), and $\mathrm{CdTe}$ (edge Te). The dashed lines are the best-fitting Einstein models. 


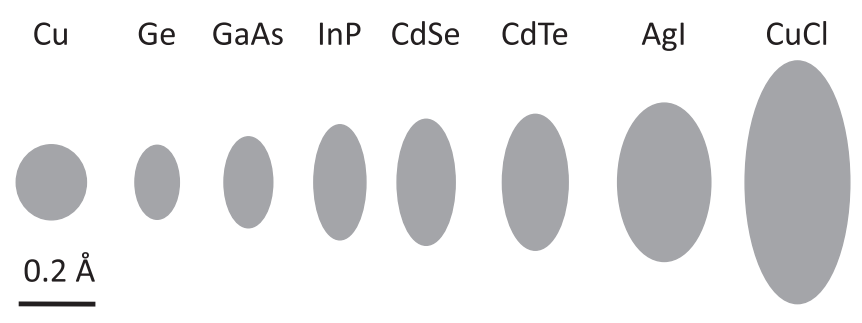

FIG. 9. In-scale two-dimensional projections of the ellipsoids of relative displacements at $T=300 \mathrm{~K}$. The bond direction is horizontal.

distribution of relative displacements (if cylindrical symmetry is assumed around the bond direction). The standard deviations $\sigma_{\|}=\left[\left\langle\Delta u_{\|}^{2}\right\rangle\right]^{1 / 2}$ and $\sigma_{\perp}=\left[\left\langle\Delta u_{\perp}^{2}\right\rangle / 2\right]^{1 / 2}$ measure the axes of the ellipsoids, which can be visually represented in the real space (Fig. 9). It turns out that the ellipsoids of relative vibrations are anisotropic (disc-shaped). The degree of anisotropy can be measured by an anisotropy parameter ${ }^{37} \xi=\left(k_{||} / k_{\perp}\right)^{1 / 2}$, which corresponds to the high-temperature asymptotic ratio $\sigma_{\perp} / \sigma_{\|}$and whose values are listed in Table I.

In copper and in the tetrahedral semiconductors here considered, the vibrations of single atoms, measured by Bragg diffraction, are isotropic, for symmetry reasons. In copper, even the relative vibrations characterised by the MSRD are nearly isotropic, the anisotropy parameter being $\xi=1.08$. In tetrahedral semiconductors, the anisotropy parameter is $\xi=1.71$ for $\mathrm{Ge}$ and exhibits an average positive trend as a function of ionicity, up to the value 2.32 for $\mathrm{CuCl}$. A more neat correlation can be found with the mass ratios $M_{h} / M_{l}$ between heavier and lighter atoms: the larger the mass ratio, the larger the anisotropy. ${ }^{62}$

The anisotropy of relative vibrations can be explained in terms of the different correlation of atomic vibrations parallel and perpendicular to the bond direction. ${ }^{37,104}$ The parallel correlation is stronger than the perpendicular correlation and quite independent of ionicity, while the perpendicular correlation exhibits an average decreasing trend. ${ }^{62}$ For copper, the parallel correlation is only slightly larger than the perpendicular correlation. The different behaviour of the systems here considered can be qualitatively explained in terms of the difference between the metallic bond of copper and the totally or partially covalent bonds of semiconductors. In tetrahedral semiconductors, the open structure facilitates the perpendicular relative vibrations of neighbouring atoms, notwithstanding the relative bond rigidity, which anyway decreases at the increase of ionicity. The weak resistance of the metallic bond against bending is compensated by the close atomic packing of the fcc structure.

In crystals of lower local symmetry than copper and tetrahedral semiconductors, the vibrations of atoms that are linearly coordinated to only two nearest-neighbours are generally anisotropic. Examples studied by EXAFS are some crystals with the cuprite, ${ }^{53}$ delafossite, ${ }^{55}$ perovskite, ${ }^{54}$ or anti-perovskite structures. ${ }^{104}$ Some relevant parameters for these crystals are shown in Table II. In these cases, the anisotropy of relative vibrations was found larger than the anisotropy of single atom vibrations, again corresponding to a smaller correlation in the perpendicular than in the parallel direction.
TABLE II. MSRD parameters for linearly coordinated absorber atoms in some crystals with layered or framework structures.

\begin{tabular}{lccc}
\hline \hline & $k_{0}=k_{\|}\left(\mathrm{eV} / \AA^{2}\right)$ & $k_{\perp}\left(\mathrm{eV} / \AA^{2}\right)$ & $\left(k_{\|} / k_{\perp}\right)^{1 / 2}$ \\
\hline $\mathrm{CuLaO}_{2}{ }^{55}$ & 15.5 & 2.6 & 2.4 \\
$\mathrm{CuScO}_{2}^{55}$ & 24.2 & 1.0 & 4.9 \\
$\mathrm{Cu}_{2} \mathrm{O}^{53}$ & 11.6 & 2.9 & 2.0 \\
$\mathrm{Ag}_{2} \mathrm{O}^{53}$ & 5.9 & 0.5 & 3.4 \\
$\mathrm{ReO}_{3}{ }^{54,104}$ & & & 1.6 \\
$\mathrm{Cu}_{3} \mathrm{~N}^{104}$ & 9.25 & 2.0 & 2.2 \\
\hline \hline
\end{tabular}

The degree of correlation has been generally evaluated by comparing the MSRDs with the uncorrelated single-atom mean square displacements (MSDs) obtained from diffraction experiments, when available. The possibility of calculating the atomic MSDs by a Reverse Monte Carlo simulation with evolutionary algorithm applied to the EXAFS analysis has recently led to evaluate the correlation in some perovskite structures without performing diffraction experiments. ${ }^{104}$

Little attention has been paid up to now to the possibility of obtaining original information on physical phenomena from the knowledge of the three-dimensional MSRD. Let us propose here two possible applications: the local origin of NTE and the ionic movements leading to the superionic transition.

\section{B. MSRD and negative thermal expansion}

As already observed, the overall lattice thermal expansion is the result of two contributions, a positive bond expansion, directly measured by the first EXAFS cumulant, and a negative contribution due to tension effects. ${ }^{44,85}$ The perpendicular MSRD is a measure of the tension effects and can be correlated with the strength of the lattice NTE, say with the value of the negative expansion coefficient and the temperature interval where NTE is present. ${ }^{62}$

The semiconductors with the zincblende structure here considered exhibit lattice NTE in a restricted lowtemperature interval, whose strength increases when the ionicity increases. ${ }^{83}$ Other NTE crystals have been recently investigated by EXAFS: $\mathrm{Cu}_{2} \mathrm{O}$ and $\mathrm{Ag}_{2} \mathrm{O}$ (cuprite structure) ${ }^{53}$ and $\mathrm{CuScO}_{2}$ and $\mathrm{CuLaO}_{2}$ (delafossite structure), ${ }^{55}$ where the metal atom $(\mathrm{Cu}$ or $\mathrm{Ag})$ is linearly coordinated to two oxygen ions. It is instructive to stress similarities and differences among the different systems.

The lattice thermal expansion is isotropic in the cubic zincblende and cuprite structures; it is anisotropic in the layered delafossite structure, where it is negative along the $c$ axis and positive in the perpendicular plane. The vibrations of the $\mathrm{Cu}$ or $\mathrm{Ag}$ ions are anisotropic in the cuprite and delafossite structures. Atomic vibrations perpendicular to a linear bond are the simplest example of tension effect leading to NTE contributions. The vibrations of the tetrahedrally coordinated single atoms in the zincblende structure are instead isotropic. The MSRD thermal ellipsoids are always anisotropic, even for the zincblende structures. The relative anisotropy measured by EXAFS for the $\mathrm{Cu}-\mathrm{O}$ and $\mathrm{Ag}-\mathrm{O}$ pairs in the cuprite and delafossite structures is larger than the anisotropy of single atomic vibrations. One can conclude that the relative vibrational anisotropy is a more general property to be 
connected to NTE than the anisotropy of single atoms vibrations.

The bond thermal expansion measured by EXAFS is positive at all temperatures for all systems up to now considered; besides, to a stronger lattice NTE, it corresponds a larger positive bond expansion. Within each family of iso-structural compounds, the stronger is the NTE, the smaller is the value of both parallel and perpendicular effective force constants and the larger is the anisotropy parameter $\xi=\left(k_{||} / k_{\perp}\right)^{1 / 2}$.

Bond stretching and tension effects are separately quantified in EXAFS analyses, in terms of first cumulant and perpendicular MSRD, respectively. They are however in some way correlated: when the ionicity of the bond increases, its stiffness against bending is reduced ( $k_{\perp}$ decreases) so that the perpendicular MSRD becomes larger. If the structure is relatively open, as in tetrahedral semiconductors, larger vibrations perpendicular to the bond make possible larger parallel vibrations too (smaller $k_{\|}$) and as a consequence a stronger bond expansion.

A particular intriguing case is represented by $\mathrm{ReO}_{3}$, whose perovskite-like structure is in principle the ideal support for rigid unit modes (RUM) leading to lattice NTE. Actually, while the iso-structural compound $\mathrm{ScF}_{3}$ is affected by a strong NTE from 0 to $600 \mathrm{~K},{ }^{105}$ a comparatively much smaller variation of the lattice parameter has been found for $\mathrm{ReO}_{3} \cdot{ }^{54,106}$ EXAFS measurements on both $\mathrm{ReO}_{3}$ and $\mathrm{ScF}_{3}{ }^{54,107}$ revealed a large intensity of $\mathrm{Re}-\mathrm{O}$ and $\mathrm{Sc}-\mathrm{F}$ relative vibrations perpendicular to the bond, as expected in the presence of RUMs inducing relative librations of the octahedra around the connecting vertices. The lack of significant NTE in $\mathrm{ReO}_{3}$ was tentatively explained hypothesising a distorted configuration of the $\mathrm{ReO}_{6}$ octahedra at low temperatures. In this situation, according to the model of Ref. 44, the librations of the octahedra around the distorted configuration would induce a positive lattice expansion as long as they are not sufficient to cross the undistorted configuration.

\section{MSRD and superionic crystals}

Let us consider in more detail the two systems characterised by the most intense parallel and perpendicular relative vibrations in Table I, $\mathrm{AgI}$ and $\mathrm{CuCl}$. Both systems have been extensively studied for their properties of ionic conduction. They are characterised by a first-order phase transition from a normal to a superionic phase, where the cations freely move within the anion sublattice. In AgI, the phase transition from the normal $\beta$ phase (wurtzite structure) to the superionic $\alpha$ phase (bcc Iodine sublattice) occurs at $T=420 \mathrm{~K}$, where the ionic conductivity increases abruptly by a factor $10^{4}$. $\mathrm{In} \mathrm{CuCl}$, the phase transition from the normal $\gamma$ phase (zincblende structure) to the superionic $\beta$ phase (hcp $\mathrm{Cl}$ sublattice) occurs at $T=695 \mathrm{~K}$; the ionic conductivity in $\mathrm{CuCl}$ increases however more rapidly than exponential already in the normal phase, so that the jump at the phase transition is relatively small.

In a pioneering study, ${ }^{108}$ Boyce et al. obtained a satisfactory description of the one-dimensional EXAFS distribution of distances with an excluded-volume model, where the anions oscillate around their lattice sites and only cation-anion interactions are considered: the cation density is zero within a distance $r_{\mathrm{excl}}=r_{c}+r_{a}$ from the anion sites $\left(r_{a}\right.$ and $r_{c}$ are the anion and cation radii, respectively), it is a constant different from zero elsewhere. According to the EXAFS analysis, when the temperature increases, the excluded volume decreases, due to the anion thermal agitation, while the distance $r_{\text {face }}$ of the anions from the centres of the tetrahedral faces increases. In AgI, in the $\beta$ phase $r_{\text {excl }}>r_{\text {face }}$ and the possibility of cations leaving the tetrahedral cage is low; at the $\beta \rightarrow \alpha$ phase transition, $r_{\text {face }}$ increases abruptly and becomes significantly larger than $r_{\text {excl }}$, so that cations can easily leave the tetrahedral cage, giving rise to the superionic regime. $\mathrm{In} \mathrm{CuCl}$, the difference $r_{\text {excl }}-r_{\text {face }}$ is much smaller than in $\mathrm{AgI}$ at room temperature and becomes negative at temperatures much lower than the transition temperature.

The cumulant analysis of $\beta$-AgI EXAFS has been shown to be consistent with the one-dimensional distribution of distances of the excluded-volume model, provided the convergence properties of the cumulant series are properly taken into account above $300 \mathrm{~K} .^{12,75}$ Relatively minor attention has been paid, to our knowledge, to a cumulant approach to the superionic transition of AgI; two independent works ${ }^{109,110}$ agree in quoting a slight abrupt increase of the second cumulant and little or no significant variations of the odd cumulants.

A possible connection between the perpendicular MSRD in $\beta$-AgI and the superionic transition was qualitatively hypothesised in Ref. 111. The comparison of the threedimensional distributions of vector distances, evaluated here at room temperature (Fig. 9), allows one to gain a deeper quantitative understanding of the different behaviour of $\mathrm{AgI}$ and $\mathrm{CuCl}$ and gives some hints on the vibrational origin of the cation mobility. The relative extent of the perpendicular vibrations $\sigma_{\perp} /\langle r\rangle$ measures the probability of the cation to approach the faces of the tetrahedral cage of anions and possibly to escape the cage, thanks to the relatively large extent of parallel vibrations $\sigma_{\|} /\langle r\rangle$ (see numerical values in Table I). The different behaviour of $\mathrm{AgI}$ and $\mathrm{CuCl}$ can be connected to the different extent of the ellipsoids of Fig. 9 for the two systems, the one of $\mathrm{AgI}$ not yet larger enough to guarantee a significant ionic conduction in the normal phase, the one of $\mathrm{CuCl}$ sufficiently large to explain the large increase of the ionic conduction already at relatively low temperatures. These considerations are consistent with the results of Boyce et al., where at room temperature the difference $r_{\text {excl }}-r_{\text {face }}$ is about $0.11 \AA$ for $\mathrm{AgI}$ and about $0.04 \AA$ for $\mathrm{CuCl}$.

\section{CONCLUSIONS}

For relatively weak vibrational disorder in crystals, the distribution of nearest-neighbour distances can be characterised by the numerical values of its cumulants. The leading cumulants are obtained from the analysis of the EXAFS signal and give direct information on the bond expansion and on the width and shape of the distribution. The nearest-neighbour distribution is intrinsically asymmetric, so that the mean value (bond distance) is different from the mode; neglecting the third cumulant in the data analysis, say relying on the gaussian approximation, can lead to significantly wrong values of bond length and bond expansion. 
Once the leading cumulants have been determined, one can reconstruct the one-dimensional asymmetric distribution of bond lengths, which can be compared with the distribution directly obtained by the Fourier transform of total scattering data, if available, allowing a direct comparison between the two techniques. It should be noticed that the artefacts of Fourier transforms are eliminated or drastically reduced in the EXAFS data analysis and do not influence the values of cumulants and the shape of the reconstructed distribution.

The knowledge of the numerical values of the leading cumulants, and possibly their temperature dependence, allows a deeper quantitative understanding of the difference between the local properties probed by a correlation-sensitive technique such as EXAFS and the average properties probed by Bragg scattering than the sole knowledge of the distribution. A significant example is the possibility of evaluating the temperature dependence of the coefficient of bond thermal expansion, to be compared with the coefficient of lattice expansion.

The effective pair potential of the nearest-neighbour pairs is anharmonic; its force constants can be evaluated from the temperature dependence of cumulants. Different and non equivalent measures of anharmonicity can be defined in order to compare the behaviour of different systems: the third cumulant, the ratio of the third- to second-order force constants, the asymmetry coefficient of the distribution of distances, the coefficient of bond thermal expansion. The anharmonicity of the effective pair potential should not be confused with the anharmonicity of the crystal potential.

The comparison of the EXAFS data with the Bragg diffraction data allows the evaluation of the perpendicular MSRDs and of the correlation effects in atomic vibrations parallel and perpendicular to the bond direction. From the knowledge of both parallel and perpendicular MSRDs, it is possible to reconstruct a three-dimensional ellipsoid of relative vibrations, which turns out to be anisotropic even when the vibrations of single atoms are isotropic. The extent of perpendicular MSRD and the anisotropy of relative vibrations can be connected to the lattice negative thermal expansion and give original insights on the ionic mobility and on the onset of phase instabilities.

The present results are intended to stimulate further work on different systems, in order to thoroughly compare the nearest-neighbour distributions obtainable from EXAFS and from total scattering, as well as to quantitatively exploit the three-dimensional distributions of bond distances.

\section{ACKNOWLEDGMENTS}

Most results here reviewed are based on experiments performed in cooperation with a number of people, to which the authors are indebted: G. Dalba, F. Rocca, N. Abd el All, S. Ahmed, M. Vaccari, A. Sanson, J. Purans, A. Kuzmin, D. Diop, and B. Thiodjio Sendja. Part of the present work originated during a one-month stay of T. Miyanaga at the Physics Department of the University of Trento in 2013. C. Schnohr is warmly thanked for communicating numerical data of InP and for precious comments on the manuscript.
${ }^{1} \mathrm{~J}$. Als-Nielsen and D. McMorrow, Elements of Modern X-Ray Physics (John Wiley \& Sons, Chichester, UK, 2011).

${ }^{2}$ S. Calvin, XAFS for Everyone (CRC Press, Taylor \& Francis, 2013).

${ }^{3} X$-Ray Absorption and X-Ray Emission Spectroscopy, Theory and Applications, edited by J. A. van Bokhoven and C. Lamberti (John Wiley \& Sons, 2016).

${ }^{4}$ P. Eisenberger and G. S. Brown, Solid State Commun. 29, 481 (1979).

${ }^{5}$ J. B. Boyce, T. M. Hayes, W. Stutius, and J. C. Mikkelsen, Jr., Phys. Rev. Lett. 38, 1362 (1977).

${ }^{6}$ E. D. Crozier and A. J. Seary, Can. J. Phys. 58, 1388 (1980).

${ }^{7}$ E. D. Crozier, J. J. Rehr, and R. Ingalls, in X-Ray Absorption, edited by D. C. Koningsberger and R. Prins (John Wiley \& Sons, New York, 1988), Chap. 9, pp. 373-442.

${ }^{8}$ A. Filipponi and A. Di Cicco, Phys. Rev. B 51, 12322 (1995).

${ }^{9}$ H. Cramér, Mathematical Methods of Statistics (Princeton University Press, 1966).

${ }^{10}$ G. Bunker, Nucl. Instrum. Methods Phys. Res. 207, 437 (1983).

${ }^{11}$ T. Fujikawa and T. Miyanaga, J. Phys. Soc. Jpn. 62, 4108 (1993).

${ }^{12}$ G. Dalba, P. Fornasini, and F. Rocca, Phys. Rev. B 47, 8502 (1993).

${ }^{13}$ J. M. Tranquada and R. Ingalls, Phys. Rev. B 28, 3520 (1983).

${ }^{14}$ G. Dalba, P. Fornasini, R. Grisenti, and J. Purans, Phys. Rev. Lett. 82, 4240 (1999).

15 J. M. Tranquada and R. Ingalls, Phys. Rev. B 34, 4267 (1986).

${ }^{16}$ A. San-Miguel, A. Polian, M. Gauthier, and J. P. Itié, Phys. Rev. B 48, 8683 (1993).

${ }^{17}$ G. Dalba, P. Fornasini, D. Diop, M. Grazioli, and F. Rocca, J. Non-Cryst. Solids 164-166, 159 (1993).

${ }^{18}$ C. S. Schnohr, P. Kluth, L. L. Araujo, D. J. Sprouster, A. P. Byrne, G. J. Foran, and M. C. Ridgway, Phys. Rev. B 79, 195203 (2009).

${ }^{19}$ D. S. Yang, D. R. Fazzini, T. I. Morrison, L. Tröger, and G. Bunker, J. NonCryst. Solids 210, 275 (1997).

${ }^{20}$ A. Filipponi, J. Phys.: Condens. Matter 13, R23 (2001).

${ }^{21}$ E. A. Stern, P. Livins, and Z. Zhang, Phys. Rev. B 43, 8850 (1991).

22 A. Filipponi, J. Phys.: Condens. Matter 6, 8415 (1994).

${ }^{23}$ A. D. Cicco, A. Trapananti, S. Faggioni, and A. Filipponi, Phys. Rev. Lett. 91, 135505 (2003).

${ }^{24}$ B. S. Clausen and J. K. Nørskov, Top. Catal. 10, 221 (2000).

${ }^{25} \mathrm{G}$. Agostini, A. Piovano, L. Bertinetti, R. Pellegrini, G. Leofanti, E. Groppo, and C. Lamberti, J. Phys. Chem. C 118, 4085 (2014).

${ }^{26} \mathrm{G}$. Agostini, R. Grisenti, C. Lamberti, A. Piovano, and P. Fornasini, J. Phys.: Conf. Ser. 430, 012031 (2013).

${ }^{27}$ A. Yevick and A. I. Frenkel, Phys. Rev. B 81, 115451 (2010).

${ }^{28}$ S. T. Chill, R. M. Anderson, D. F. Yancey, A. I. Frenkel, R. M. Crooks, and G. Henkelman, ACS Nano 9, 4036 (2015).

${ }^{29}$ Y. A. Babanov, V. V. Vasin, A. L. Ageev, and N. V. Ershov, Phys. Status Solidi B 105, 747 (1981).

${ }^{30}$ D. S. Yang and G. Bunker, Phys. Rev. B 54, 3169 (1996).

${ }^{31}$ A. Kuzmin and Y. Purans, Radiat. Meas. 33, 583 (2001).

${ }^{32}$ P. D'Angelo, A. Di Nola, A. Filipponi, N. V. Pavel, and D. Roccatano, J. Chem. Phys. 100, 985 (1994).

${ }^{33}$ A. Kuzmin and R. A. Evarestov, J. Phys.: Condens. Matter 21, 055401 (2009).

${ }^{34}$ S. W. T. Price, N. Zonias, C. Skylaris, T. I. Hyde, B. Ravel, and A. E. Russell, Phys. Rev. B 85, 075439 (2012).

${ }^{35}$ S. J. Gurman and R. L. McGreevy, J. Phys.: Condens. Matter 2, 9463 (1990).

36J. Timoshenko and A. I. Frenkel, Catal. Today 280, 274 (2017).

${ }^{37}$ P. Fornasini and R. Grisenti, J. Synchrotron Radiat. 22, 1242 (2015).

${ }^{38}$ P. Fornasini, S. a Beccara, G. Dalba, R. Grisenti, A. Sanson, M. Vaccari, and F. Rocca, Phys. Rev. B 70, 174301 (2004).

${ }^{39}$ S. a Beccara, G. Dalba, P. Fornasini, R. Grisenti, F. Pederiva, A. Sanson, D. Diop, and F. Rocca, Phys. Rev. B 68, 140301 (2003).

${ }^{40}$ F. D. Vila, J. J. Rehr, H. H. Rossner, and H. J. Krappe, Phys. Rev. B 76, 014301 (2007).

${ }^{41}$ A. Sanson, Phys. Rev. B 81, 012304 (2010).

${ }^{42}$ W. R. Busing and H. A. Levy, Acta Crystallogr. 17, 142 (1964).

${ }^{43}$ B. T. M. Willis and A. W. Pryor, Thermal Vibrations in Crystallography (Cambridge University Press, 1975).

${ }^{44}$ G. D. Barrera, J. A. Bruno, T. H. K. Barron, and N. L. Allan, J. Phys.: Condens. Matter 17, R217 (2005).

${ }^{45}$ T. Egami and S. J. L. Billinge, Underneath the Bragg Peaks: Structural Analysis of Complex Materials (Pergamon, Amsterdam, 2003).

${ }^{46}$ M. G. Tucker, M. T. Dove, and D. A. Keen, J. Phys.: Condens. Matter 12, L425 (2000). 
${ }^{47}$ M. G. Tucker, A. L. Goodwin, M. T. Dove, D. A. Keen, S. A. Wells, and J. S. O. Evans, Phys. Rev. Lett. 95, 255501 (2005).

${ }^{48}$ K. W. Chapman, P. J. Chupas, and C. J. Kepert, J. Am. Chem. Soc. 127, 15630 (2005).

${ }^{49}$ M. Dapiaggi, H. Kim, E. S. Božin, S. J. L. Billinge, and G. Artioli, J. Phys. Chem. Solids 69, 2182 (2008).

${ }^{50}$ K. W. Chapman and P. J. Chupas, Chem. Mater. 21, 425 (2009).

${ }^{51}$ N. Abd el All, G. Dalba, D. Diop, P. Fornasini, R. Grisenti, O. Mathon, F. Rocca, B. Thiodjio Sendja, and M. Vaccari, J. Phys.: Condens. Matter 24, 115403 (2012).

${ }^{52}$ S. I. Ahmed, G. Aquilanti, N. Novello, L. Olivi, R. Grisenti, and P. Fornasini, J. Chem. Phys. 139, 164512 (2013).

${ }^{53}$ A. Sanson, F. Rocca, G. Dalba, P. Fornasini, R. Grisenti, M. Dapiaggi, and G. Artioli, Phys. Rev. B 73, 214305 (2006).

${ }^{54}$ J. Purans, P. Fornasini, S. E. Ali, G. Dalba, A. Kuzmin, and F. Rocca, Phys. Rev. B 92, 014302 (2015).

${ }^{55}$ S. I. Ahmed, G. Dalba, P. Fornasini, M. Vaccari, F. Rocca, A. Sanson, J. Li, and A. W. Sleight, Phys. Rev. B 79, 104302 (2009).

${ }^{56}$ Y. S. Touloukian, R. K. Kirby, R. E. Taylor, and P. D. Desai, Thermophysical Properties of Matter (Plenum, New York, 1977), Vol. 13.

${ }^{57}$ P. Fornasini and R. Grisenti, J. Chem. Phys. 141, 164503 (2014).

${ }^{58}$ P. Fornasini, J. Phys.: Condens. Matter 13, 7859 (2001).

${ }^{59}$ G. Beni and P. M. Platzman, Phys. Rev. B 14, 1514 (1976).

${ }^{60}$ V. Krayzman, I. Levin, J. C. Woicik, T. Proffen, T. A. Vanderah, and M. G. Tucker, J. Appl. Crystallogr. 42, 867 (2009).

${ }^{61}$ A. H. Clark, H. R. Marchbank, T. I. Hyde, H. Y. Playford, M. G. Tucker, and G. Sankar, Phys. Scr. 92, 034002 (2017).

${ }^{62} \mathrm{P}$. Fornasini, in X-Ray Absorption Spectroscopy of Semiconductors, edited by C. S. Schnohr and M. C. Ridgway (Springer, New York, 2015), Chap. 6, pp. 127-141.

${ }^{63}$ J. Purans, N. D. Afify, G. Dalba, R. Grisenti, S. De Panfilis, A. Kuzmin, V. I. Ozhogin, F. Rocca, A. Sanson, S. I. Tiutiunnikov et al., Phys. Rev. Lett. 100, 055901 (2008).

${ }^{64}$ J. Purans, J. Timoshenko, A. Kuzmin, G. Dalba, P. Fornasini, R. Grisenti, N. D. Afify, F. Rocca, S. D. Panfilis, I. Ozhogin et al., J. Phys.: Conf. Ser. 190, 012063 (2009).

${ }^{65}$ D. A. Keen, J. Appl. Crystallogr. 34, 172 (2001).

${ }^{66}$ C. S. Schnohr, L. J. Araujo, and M. C. Ridgway, J. Phys. Soc. Jpn. 83, 094602 (2014).

${ }^{67}$ M. Vaccari, Ph.D. thesis, University of Trento, Italy, 2006.

${ }^{68}$ M. Vaccari, R. Grisenti, P. Fornasini, F. Rocca, and A. Sanson, Phys. Rev. B 75, 184307 (2007).

${ }^{69}$ J. Freund, R. Ingalls, and E. D. Crozier, Phys. Rev. B 39, 12537 (1989).

${ }^{70}$ J. J. Rehr and R. C. Albers, Rev. Mod. Phys. 72, 621 (2000).

${ }^{71}$ M. Newville, J. Synchrotron Radiat. 8, 322 (2001).

${ }^{72} \mathrm{See}$ http://feffproject.org for FEFF, Project home page, 2012.

${ }^{73}$ See http://gnxas.unicam.it/ for GNXAS, Home page, 2015.

${ }^{74}$ See http://bruceravel.github.io/demeter/ for Demeter, Home page, 2015.

${ }^{75}$ G. Dalba, P. Fornasini, R. Gotter, and F. Rocca, Phys. Rev. B 52, 149 (1995).

${ }^{76}$ G. Dalba, P. Fornasini, M. Grazioli, and F. Rocca, Phys. Rev. B 52, 11034 (1995).
${ }^{77}$ G. Dalba, P. Fornasini, R. Grisenti, D. Pasqualini, D. Diop, and F. Monti, Phys. Rev. B 58, 4793 (1998).

${ }^{78}$ G. Dalba, P. Fornasini, R. Grisenti, F. Rocca, I. Chambouleyron, and C. F. O. Graeff, J. Phys.: Condens. Matter 9, 5875 (1997).

${ }^{79}$ Y. Ma and E. A. Stern, Phys. Rev. B 38, 3754 (1988).

${ }^{80}$ E. A. Stern, Y. Ma, O. Hanske-Petitpierre, and C. E. Bouldin, Phys. Rev. B 46, 687 (1992).

${ }^{81}$ I. Ono, T. Yokoyama, H. Sato, K. Kaneyuki, and T. Ohta, Jpn. J. Appl. Phys., Part 1 32(S2), 83 (1993).

${ }^{82}$ N. Abd el All, B. Thiodjio Sendja, R. Grisenti, F. Rocca, D. Diop, O. Mathon, S. Pascarelli, and P. Fornasini, J. Synchrotron Radiat. 20, 603 (2013).

${ }^{83}$ T. F. Smith and G. K. White, J. Phys. C: Solid State Phys. 8, 2031 (1975).

${ }^{84}$ E. A. Stern, J. Phys. IV France 7, C2-137 (1997).

${ }^{85}$ V. Heine, P. R. L. Welche, and M. T. Dove, J. Am. Ceram. Soc. 82, 1793 (1999).

${ }^{86}$ S. I. Novikova, Sov. Phys. - Solid State 2, 2087 (1961).

${ }^{87}$ N. W. Aschroft and N. D. Mermin, Solid State Physics (Thomson Brooks/Cole, 1976).

${ }^{88}$ H. M. Kagaya and T. Soma, Phys. Status Solidi B 138, K13 (1986).

${ }^{89}$ R. P. Gupta, P. K. Sharma, and S. Pal, Phys. Status Solidi B 18, 119 (1966).

${ }^{90}$ J. K. Baria and A. R. Jani, Physica B 328, 317 (2003).

${ }^{91}$ A. I. Frenkel and J. J. Rehr, Phys. Rev. B 48, 585 (1993).

${ }^{92}$ T. Yokoyama, J. Synchrotron Radiat. 6, 323 (1999).

${ }^{93}$ J. Mustre de Leon, S. D. Conradson, I. Batistić, A. R. Bishop, I. D. Raistrick, M. C. Aronson, and F. H. Garzon, Phys. Rev. B 45, 2447 (1992).

${ }^{94}$ T. Yokoyama and K. Eguchi, Phys. Rev. Lett. 107, 065901 (2011).

${ }^{95}$ S. a Beccara and P. Fornasini, Phys. Rev. B 77, 172304 (2008).

${ }^{96}$ A. Sanson, J. Phys.: Condens. Matter 23, 315401 (2011).

${ }^{97}$ N. Van Hung and P. Fornasini, J. Phys. Soc. Jpn. 76, 084601 (2007).

${ }^{98}$ N. Van Hung and J. J. Rehr, Phys. Rev. B 56, 43 (1997).

${ }^{99}$ T. H. K. Barron, Ann. Phys. 1, 77 (1957).

${ }^{100}$ G. Leibfried and W. Ludwig, Solid State Phys. 12, 275 (1961)

${ }^{101}$ M. Vaccari and P. Fornasini, J. Synchrotron Radiat. 13, 321 (2006).

${ }^{102}$ P. Fornasini, F. Monti, and A. Sanson, J. Synchrotron Radiat. 8, 1214 (2001).

${ }^{103}$ I. K. Jeong, T. Proffen, F. Mohiuddin-Jacobs, and S. J. L. Billinge, J. Phys. Chem. A 103, 921 (1999).

${ }^{104}$ J. Timoshenko, A. Anspoks, A. Kalinko, and A. Kuzmin, Acta Mater. 129, 61 (2017).

${ }^{105}$ B. K. Greve, K. L. Martin, P. L. Lee, P. J. Chupas, K. W. Chapman, and A. P. Wilkinson, J. Am. Chem. Soc. 132, 15496-15498 (2010).

${ }^{106}$ M. Dapiaggi and A. N. Fitch, J. Appl. Crystallogr. 42, 253 (2009).

${ }^{107}$ J. Purans, S. Piskunov, D. Bocharov, A. Kalinko, A. Kuzmin, S. E. Ali, and F. Rocca, J. Phys.: Conf. Ser. 712, 012013 (2016).

${ }^{108}$ J. B. Boyce, T. M. Hayes, and J. C. Mikkelsen, Jr., Phys. Rev. B 23, 2876 (1981).

${ }^{109}$ A. Yoshiasa, H. Maeda, T. Ishii, and K. Koto, Solid State Ionics 40-41, 341 (1990).

${ }^{110}$ G. Dalba, P. Fornasini, R. Gotter, S. Cozzini, M. Ronchetti, and F. Rocca, Solid State Ionics 69, 13 (1994).

${ }^{111}$ P. Fornasini, AIP Conf. Proc. 882, 94-98 (2007). 